\title{
Low-Dose Spiral CT
}

National Cancer Institute

\section{Source}

National Cancer Institute. Low-Dose Spiral CT. NCI Thesaurus. Code C20094.

A type of computed tomography whereby a low dose of X-ray modality is used to image certain organs i.e. the lungs. 